\title{
The Impact of Triage for Atypical Squamous Cells of Undetermined Significance with Human Papillomavirus Testing in Cervical Cancer Screening in Japan
}

\author{
Hiroyuki Fujiwara ${ }^{1}$, Mitsuaki Suzuki ${ }^{2 *}$, Hiroyuki Morisawa ${ }^{1}$, Masaaki Sayama ${ }^{3}$, \\ Kouzo Kimura ${ }^{4}$
}

\begin{abstract}
Background: One of the features of cervical cancer screening using the combination of cytology and human papillomavirus (HPV) testing is the triage for atypical squamous cells of undetermined significance (ASC-US). The effectiveness of the triage has been recognized widely. However, there are few reports evaluating this triage process in Japan. Material and Methods: We retrospectively examined the results of cytology and HPV co-testing for cervical cancer screening in the Oyama area of Tochigi Prefecture between 2012 and 2014. Women who were ASC-US/HPV positive and had cytologic abnormalities [low-grade squamous intraepithelial lesions (LSIL) or worse] were examined by colposcopy. The results of the colposcopy testing were evaluated. In addition, we also examined the results of those who underwent co-testing a year after a ASC-US/HPV-negative result. Results: A total of 21,342 women received their first screening test during the study period, with 542 (2.5\%) found to have ASC-US. Of the ASC-US-positive women, 289 (53.3\%) were also HPV positive. The prevalence of CIN + (cervical intraepithelial neoplasia or higher) in the ASC-US/HPV-positive group was $63.2 \%$, with $81.8 \%, 16.4 \%$ and $4.8 \%$. showing CIN 1, CIN 2 and CIN 3+, respectively. The prevalence of CIN + in the LSIL group was $66.8 \%$, with the majority having a low risk CIN 1 (76.6\%) compared to CIN $2(18.6 \%)$, and CIN $3+(4.8 \%)$. No significant difference was observed between the LSIL and ASC-US/ HPV-positive groups. The prevalence of women diagnosed with CIN in the ASC-US/HPV-negative group, following co-testing a year after colposcopy was low (3\%). Conclusions: The ASC-US/HPV-positive group was comparable to the LSIL group in terms of prevalence of CIN+ lesions. Furthermore, low CIN prevalence after one year in the ASCUS/HPV-negative group provides confirmation that the screening interval could be extended. The application of HPV triage (which is routine in other countries) to identify these groups would be of benefit in Japan.
\end{abstract}

Keywords: Cervical cancer screening- HPV- ASC-US

Asian Pac J Cancer Prev, 20 (1), 81-85

\section{Introduction}

Early detection of cervical cancer and dysplasia is important, not only with regard to the patient's life but for preservation of fertility. Since cytological screening for cervical cancer began in the 1960s (Anttila et al., 2004), the incidence of cervical cancer has gradually declined. However, diagnosis based on cytology has low sensitivity and reproducibility (Sasieni et al., 1996; Cuzick et al., 2006).

It has been reported that high-risk human papillomavirus (HPV) infection is a trigger for the onset of cervical cancer, and its detection has been used in a clinical situation and screening (Walboomers et al., 1999; IARC working Group, 2007). HPV testing has a higher sensitivity than cytology for the detection of CIN 2 and higher risk lesions, with less variation (Arbyn et al., 2006). Recently, the results of a number of randomized controlled trials showing the effectiveness of screening using cytology and HPV testing have been reported (Naucler et al., 2007; Kichener et al., 2009; Ronco et al., 2010; Rijkaart et al., 2012). The American Gynecological Society, US Preventive Services Task Force (USPSTF) and the American Cancer Society (ACS), the American Society for Colposcopy and Cervical Pathology (ASCCP) and the American Society for Clinical Pathology (ASCP) guidelines, all recommended HPV testing for cervical cancer screening (Committee on Practice Bulletins - Gynecology, 2016).

In Japan, cytology-based cervical cancer screening began in the 1960s and is still being used throughout 
the country. The Japanese Association of Obstetricians and Gynecologists published recommendations for cytology and HPV testing (Fig. 1) in 2011 (The Japanese Association of Obstetricians and Gynecologists, 2011). However, many local authorities continue to use cytology only. In Oyama area of Tochigi prefecture in Japan, combined screening using cytology and HPV testing started in 2012, so we evaluated the results of this cotesting retrospectively.

One feature of combined screening was using HPV testing as a triage for atypical squamous cells of undetermined significance (ASC-US). Similar to other countries, our recommendations indicated colposcopic examination for women who were ASC-US/HPV positive, whereas combination screening at intervals (6 to 12 months) was indicated for ASC-US/HPV-negative cases. However, there were few reports on whether this triage was useful in Japan, where there are differences in HPV prevalence, type and distribution (Sasagawa et al., 2001), low screening examination rates (Ministry of Health, Labour and Welfare, 2013) and low vaccination rates. Here, we report on the effectiveness of using HPV testing as a triage for ASC-US in Japan, based on a combined screening program being carried out in the Oyama area.

\section{Materials and Methods}

We retrospectively examined the results of screening based on cytology and HPV co-testing conducted in the Oyama area of Tochigi Prefecture (Oyama City, Shimotsuke City and Nogi Town; total population of 250,000) from 2012 to 2014 . This combined screening was launched by the local authorities in collaboration with the Department of Obstetrics and Gynecology, Jichi Medical University, to evaluate its effectiveness. The screening was performed based on cytology alone until 2011. However, from 2012, combined screening was performed at all examination facilities in the area. A Cervex brush was used for collecting cytological specimens, with liquid based cytology used for cyto-diagnosis and Hybrid capture 2 (HC2) for HPV testing. Anonymized data was provided to Jichi Medical University from each municipality. Screening was performed in accordance with the recommendations of the Japanese Association of Obstetricians and Gynecologists (Figure 1). Women who were ASC-US/HPV positive and had cytologic abnormalities [low-grade squamous intraepithelial lesions (LSIL) or worse] were examined by colposcopy. The women who were ASC-US/HPV negative underwent combined screening one year following testing. The results of colposcopic examinations for ASC-US/HPV at the first visit were compiled every fiscal year and compared with the results for those who underwent colposcopic examination based on the presence of cytological abnormalities. In addition, we examined the results from cytology and detailed colposcopic examination of those who underwent screening a year after having a ASC-US/ HPV-negative result. This study was conducted with the approval of each municipal authority as well as the Jichi Medical University Institutional Review Board.

Pearson's chi-square test with Bonferroni correction and Kruskal-Wallis test were used for multiple group comparisons, and differences with $\mathrm{P}$-values $<0.05 / 3$ were considered to indicate a statistically significant difference.

\section{Results}

A total of 21,342 women underwent their first cervical screening between 2012 and 2014. Table 1 shows the annual trends in the screening results. The incidence of ASC-US was around 3\%, consistent with the global recommendation that a rate of $5 \%$ or less was expected. Of the 542 cases with ASC-US, 289 (53.3\%) were HPV positive and 261 women (90\%) underwent colposcopic examination. CIN 1 to CIN $3+$ cases were observed in 165 (63.2\%) women, with 130 who were CIN 1 (78.8\%), 27 who were CIN 2 (16.4\%), and 8 who were CIN $3+(4.8 \%)$. Comparison of the results of colposcopic examinations with screening abnormalities is shown in Figure 2 and Table 2. The prevalence of CIN lesions in the LSIL group was $67.7 \%$, with CIN 1, CIN 2, and CIN 3 rates of $66.8 \%, 18.6 \%$ and $4.8 \%$, respectively. There was no statistically significant difference between the LSIL group and ASC-US/HPV-positive group. On the other hand, the prevalence of $\mathrm{CIN}+$ lesions in the high-grade squamous intraepithelial lesion (HSIL) group was $89.4 \%$, with CIN 1, CIN 2, and CIN 3+ rates of 25.7\%, 34.7\% and 39.6\%, respectively. This was significantly different from those of the ASC-US/HPV-positive and LSIL groups. The positive predictive values (PPV) for CIN $1+$ and CIN $2+$ in the ASC-US/HPV-positive group were the same as those in the LSIL group $(\mathrm{P}=0.36, \mathrm{P}=0.52$ respectively $)$ and were significantly different from those in the HSIL group ( $\mathrm{P}<0.0001, \mathrm{P}<0.0001$ respectively) .

A total of 253 women were found to be ASC-US/HPV negative, with 129 (51\%) undergoing further screening one year later. Of these, 117 cases $(91 \%)$ were negative for intraepithelial lesion or malignancy (NILM) and HPV negative (double negative) and $8(6 \%)$ were ASC-US/HPV negative. The colposcopic examination identified four cases (3\%) with cancer; one ASC-US/HPV positive, two

Table 1. Annual Trends in Cytological Screening Results (Number)

\begin{tabular}{|c|c|c|c|}
\hline Year & 2012 & 2013 & 2014 \\
\hline \multicolumn{4}{|l|}{ Screening (cytology) } \\
\hline NILM & 10,998 & 4,979 & 4,262 \\
\hline ASC-US & $316(2.70 \%)$ & $148(2.80 \%)$ & $78(1.70 \%)$ \\
\hline LISL & 171 & 122 & 110 \\
\hline HSIL & 57 & 33 & 33 \\
\hline ASC-H & 7 & 4 & 4 \\
\hline $\mathrm{SCC}$ & 1 & 0 & 1 \\
\hline AGC & 2 & 8 & 7 \\
\hline Adenocarcinoma & 1 & 0 & 0 \\
\hline Total & 11,553 & 5,294 & 4,495 \\
\hline
\end{tabular}

NILM, Negative for intraepithelial lesion or malignancy; ASC-US, Atypical squamous cells of undetermined significance; LISL, Low grade squamous intraepithelial lesion; HSIL, High grade squamous intraepithelial lesion; ASC-H, Atypical squamous cells cannot exclude HSIL; SCC, Squamous cell carcinoma; AGC, Atypical glandular cells 
Table 2. Positive Predictive Value of Screening for CIN 1 and CIN 2 Lesions. Results of screening abnormalities and colposcopic examination

\begin{tabular}{lccccccc}
\hline & Total & Normal & CIN 1 & CIN 2 & CIN 3+ & \multicolumn{2}{c}{ PPV } \\
& & & & & & CIN 1+ & CIN 2+ \\
\hline ASC-US/HPV(+) & 261 & 96 & 130 & 27 & 8 & $63.2^{*}, * *$ & $13.4^{*}, * *$ \\
LISL & 346 & 115 & 177 & 43 & 11 & 66.8 & 15.6 \\
HSIL & 113 & 12 & 26 & 35 & 40 & 89.4 & 66.4 \\
\hline
\end{tabular}

CIN, Cervical intraepitheral neoplasm; ASC-US, Atypical squamous cells of undetermined significance; LISL, Low grade squamous intraepithelial lesion; HSIL, High grade squamous intraepithelial lesion; HPV, human papillomavirus

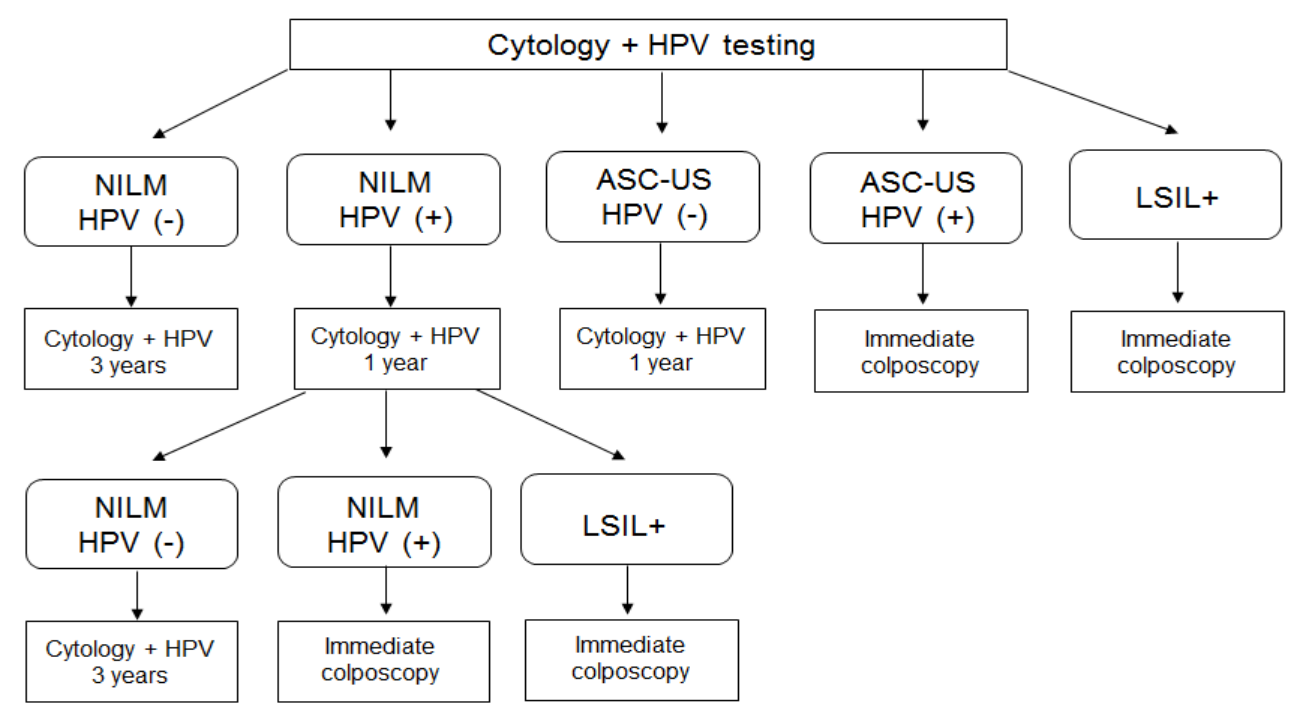

Figure 1. Protocol of Cytology-HPV Screening for Cervical Cancer in Oyama Area, Japan. NILM: Negative for intraepithelial lesion or malignancy, ASC-US: Atypical squamous cells of undetermined significance, LISL: Low grade squamous intraepithelial lesion.

LSIL/HPV positive and one HSIL/HPV positive. Detailed colposcopic examination of these four cases revealed that three had CIN 1 and one had CIN 3.

\section{Discussion}

From the results of screening, using co-testing (cytology and HPV) the ASC-US/HPV-positive group included women with CIN+ lesions at a similar level to the LSIL group. Therefore, this study validated the recommendation to perform colposcopic examination following a ASC-US/HPV-positive diagnosis in Japan. Furthermore, as the prevalence of CIN in those women screened a year following ASC-US/HPV-negative diagnosis was low, triage without immediate colposcopic examination would also be acceptable.

With regard to the handling of women with ASC-US and LSIL, the results of the ALTS (ASCUS-LSIL Triage Study; Castle et al., 2008) and other studies, have established HPV testing for use as triage. The ASCCP recommended triage using HPV testing immediately following ASC-US diagnosis or on re-examination by cytology at an interval of one year. With regard to LSIL, in cases in which HPV testing was not performed or in positive cases, colposcopic examination was recommended (Massad et al., 2012), and re-screening at an interval of one year was indicated

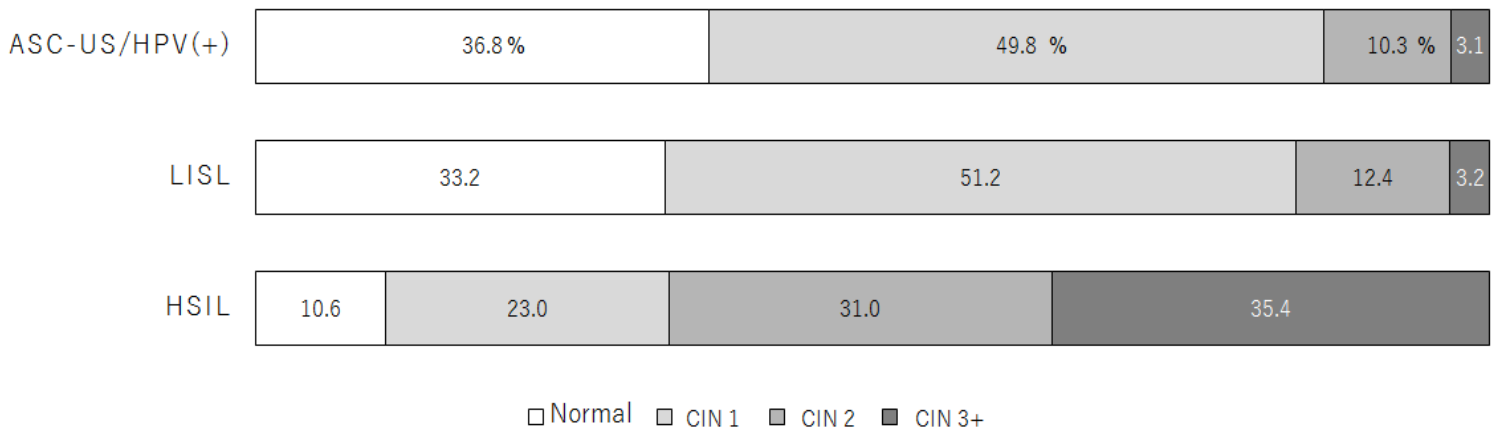

Figure 2. Proportional Distribution of Normal and CIN Lesions Following a Colposcopic Examination $( \pm$ biopsy) in ASC-US/HPV(+), LSIL and HSIL Groups. CIN:Cervical intraepitheral neoplasm, ASC-US: Atypical squamous cells of undetermined significance, LISL: Low grade squamous intraepithelial lesion, HSIL: High grade squamous intraepithelial lesion, HPV: human papillomavirus. 
for HPV-negative cases. However, although HPV testing was effective as triage for ASC-US, some reports have indicated that it was not recommended for LSIL in terms of its specificity (Sherman et al., 2002; Veijalainen et al., 2015). According to the Cochrane Review (Arbyn et al., 2013), triage using the HC2 test for ASC-US was more sensitive for the detection of CIN 2 than a repeat cytology test, while triage using HC2 for LSIL had low specificity. Our results that the ASC-US/HPV-positive group was equal to the LSIL-without HPV testing group support these previous findings.

The results of combined screening using cytology and HPV testing have been reported for various countries. In Italy, the accuracy of pap smears, colposcopy, and HPV testing were examined prospectively in 749 women with ASC-US, and HPV testing had the highest sensitivity, specificity and PPV (Del Mistro et al., 2010). Similarly, in Korea, the results for HPV testing as triage for ASC-US and LSIL confirmed that the screening guidelines proposed by the ASCCP in 2012 were effective (Hyun et al., 2017). In Japan, there is a report that HPV testing is useful for NILM cases predicting progression of lesions to CIN+ (Sasaki et al., 2017), however, there is no data about the triage of ASC-US with HPV testing. This study is first report to clarify the usefulness of this triage. Recently, the CITRUS, Cervical cancer screening Trial by Randomization of HPV testing intervention for Upcoming Screening trial (Morisada et al., 2017) was just started in Japan, to compare combination testing (cytology and HPV) to cytology testing alone.

The ASCCP guidelines recommended women be re-examined at an interval of three years if they were ASC-US/HPV negative (Massad et al., 2012). As with the recommendation from ASCCP, the low rate of CIN in women who were re-examined after an interval of one year in our study suggests that it was possible to extend this interval. However, the rates for cervical cancer screening in Japan are very low (Ministry of Health, Labour and Welfare, 2013) and any extension of the interval may further decrease these rates. In this study, about half $(51 \%)$ of the women screened were re-examined after one year, which was considered low. Therefore, it was considered preferable to continue employing the current one-year interval. A number of studies have attempted to ascertain the reasons for low screening rates. Whilst we studied and adopted the most effective type of consultation to encourage screening (Fujiwara et al., 2015), higher screening rates did not eventuate. Our task in the future is to increase the screening rates for cervical cancer in Japan to comparable rates seen in the US and Europe.

A limitation of this study is that contrary to general recommendations, women in their twenties were also screened. In the future, the screening results obtained for women in their twenties may be used to ascertain the appropriate age for starting combined screening.

In conclusion, women in the ASC-US/HPV-positive group were comparable to the LSIL group, with regards to the proportion who were subsequently diagnosed with CIN lesions. Therefore, the application of HPV triage to identify women at risk of developing cancerous lesions would be of benefit in Japan. Further, as the prevalence of CIN in the ASC-US/HPV-negative group was low, these women would not require immediate colposcopic examination, but screening can be employed on a yearly basis. Further study is required to clarify the appropriate number of years for this follow-up screening in Japan.

\section{Competing interests}

The authors declare that they have no competing interests. This work was supported by MEXT KAKENHI Grant Number 25460775. The funders had no role in the study design, data collection and analysis, decision to publish and preparation of the manuscript.

\section{Acknowledgments}

We appreciate the great contributions of the late Dr. Kiyoshi Hirao to this study.

\section{References}

Anttila A, Ronco G, Clifford G, et al (2004). Cervical cancer screening programmes and policies in 18 European countries. Br J Cancer, 91, 935-41.

Arbyn M, Sasieni P, Meijer CJ, et al (2006). Chapter 9: Clinical applications of HPV testing: a summary of meta-analyses. Vaccine, 24, 78-89.

Arbyn M, Roelens J, Simoens C, et al (2013). Human papillomavirus testing versus repeat cytology for triage of minor cytological cervical lesions. Cochrane Database Syst Rev, 28, CD008054.

Castle PE, Cox JT, Jeronimo J, et al (2008). An analysis of high-risk human papillomavirus DNA-negative cervical precancers in the ASCUS-LSIL Triage Study (ALTS). Obstet Gynecol, 111, 847-56.

Committee on Practice Bulletins-Gynecology (2016). Practice Bulletin No. 168: Cervical Cancer Screening and Prevention. Obstet Gynecol, 128, 111-30.

Cuzick J, Clavel C, Petry KU, et al (2006). Overview of the European and North American studies on HPV testing in primary cervical cancer screening. Int $J$ Cancer, 119, 1095-101.

Del Mistro A, Frayle-Salamanca H, Trevisan R, et al (2010). Triage of women with atypical squamous cells of undetermined significance (ASC-US): results of an Italian multicentric study. Gynecol Oncol, 117, 77-81.

Fujiwara H, Shimoda A, Ishikawa Y, et al (2015). Effect of providing risk information on undergoing cervical cancer screening: a randomized controlled trial. Arch Public Health, 73, 7.

Hyun JY, Min KJ, Yang SY, et al (2017). Atypical squamous cells of undetermined significance and low-grade squamous intraepithelial lesion triage in Korean women: Revisiting the 2012 American Society of Colposcopy and Cervical Pathology screening guidelines. Obstet Gynecol Sci, 60, 357-61.

IARC working Group on the Evaluation of Carcinogenic Risks to Humans (2007). Human Papillomaviruses. IARC Monogr Eval Carcinog Risks Hum, 90, 1-636.

Kichener HC, Almonte M, Thomson C, et al (2009). HPV testing in combination with liquid-based cytology in primary cervical screening (ARTISTIC): a randomised controlled trial. Lancet Oncol, 10, 672-82.

Massad LS, Einstein MH, Huh WK, et al (2012). 2012 Updated Consensus Guidelines for the Management of Abnormal Cervical Cancer Screening Tests and Cancer Precursors. 
ASCCP Guidelines Update, pp 1-27.

Ministry of Health, Labour and Welfare (2013). Comprehensive Survey of Living Conditions. [http://www.mhlw.go.jp/ toukei/saikin/hw/k-tyosa/k-tyosa13/d1/04.pdf].

Morisada T, Teramoto K, Takano H, et al (2017). CITRUS, cervical cancer screening trial by randomization of HPV testing intervention for upcoming screening: Design, methods and baseline data of 18,471 women. Cancer Epidemiol, 50, 60-7.

Naucler P, Ryd W, Törnberg S, et al (2007). Human papillomavirus and Papanicolaou tests to screen for cervical cancer. $N$ Eng J Med, 357, 1589-97.

Rijkaart DC, Berkhof J, Rozendaal L, et al (2012). Human papillomavirus testing for the detection of high-grade cervical intraepithelial neoplasia and cancer: final results of the POBASCAM randomised controlled trial. Lancet Oncol, 13, 78-88.

Ronco G, Giorgi-Rossi P, Carozzi F, et al (2010). Efficacy of human papillomavirus testing for the detection of invasive cervical cancers and cervical intraepithelial neoplasia: a randomised controlled trial. Lancet Oncol, 11, 249-57.

Sasagawa T, Basha W, Yamazaki H, et al (2001). High-risk and multiple human papillomavirus infections associated with cervical abnormalities in Japanese women. Cancer Epidemiol Biomarkers Prev, 10, 45-52.

Sasaki Y, Iwanari O, Arakawa I, et al (2017). Cervical cancer screening with Human Papillomavirus DNA and cytology in Japan. Int J Gynecol Cancer, 27, 523-9.

Sasieni PD, Cuzick J, Lynch-Farmery E (1996). Estimating the efficacy of screening by auditing smear histories of women with and without cervical cancer. The National Co-ordinating Network for Cervical Screening Working Group. $\mathrm{Br} J$ Cancer, 73, 1001-5.

Sherman ME, Schiffman M, Luukkaala T, et al (2002). Effects of age and human papilloma viral load on colposcopy triage: data from the randomized Atypical Squamous Cells of Undetermined Significance/Low-Grade Squamous Intraepithelial Lesion Triage Study (ALTS). J Natl Cancer Inst, 94, 102-7.

The Japanese Association of Obstetricians and Gynecologists (2011). Recommendation of cytology and HPV co-testing.

Veijalainen O, Tuomisaari S, et al (2015). High risk HPV testing in the triage of repeat ASC-US and LSIL. Acta Obstet Gynecol Scand, 94, 931-6.

Walboomers JM, Jacobs MV, Manos MM, et al (1999). Human papillomavirus is a necessary cause of invasive cervical cancer worldwide. J Pathol, 189, 12-9.

\section{c) (7) (8)}

This work is licensed under a Creative Commons AttributionNon Commercial 4.0 International License. 